\title{
The Marsh Lane Railway Viaduct: 2 years of monitoring with combined sensing and surveying technologies
}

\author{
Sinan Acikgoz ${ }^{1}$, Haris Alexakis ${ }^{2}$, Cong $\mathrm{Ye}^{2}$, Andrea Franza $^{3}$ and Matthew DeJong ${ }^{4}$ \\ ${ }^{1}$ University of Oxford, Oxford OX1 3TF, UK \\ ${ }^{2}$ University of Cambridge, Cambridge, CB2 1PZ, UK \\ ${ }^{3}$ Universidad Politécnica de Madrid, Madrid, 28040, Spain \\ ${ }^{2}$ University of California at Berkeley, Berkeley, 94720, CA, U.S.A. \\ sinan.acikgoz@eng.ox.ac.uk
}

\begin{abstract}
Marsh Lane viaduct is a typical example of a 19th century brick masonry railway arch in the UK. It frequently carries passenger trains to and from Leeds Station. This paper broadly discusses the sensing techniques and associated analysis procedures used to (i) identify the reasons for existing damage, (ii) quantify their impact on the dynamic response of the structure and (iii) measure degradation of the response over a period of one year. To identify existing damage, distortions in geometry of the structure are examined with new point cloud processing techniques. With the aid of limit analyses, these distortions are interpreted, and past support movements which may have caused the distortions are identified. Then, to measure the dynamic response of the bridge, quasidistributed fibre optic strain sensing and digital image correlation displacement measurement techniques are used. These highlight the increased dynamic response around locations of existing damage, and point out to the global mechanisms of response that could propagate damage. Continuous fibre optic strain measurements between November 2017 and 2018 are then discussed to investigate the ongoing deterioration.
\end{abstract}

Keywords: Masonry arch bridges, Point clouds, Primitive fitting, Fibre optic strain sensing, Digital image correlation

\section{Description of the Marsh Lane Viaduct}

Marsh Lane viaduct is an operational brick masonry viaduct in close proximity to Leeds central train station. It was constructed during the North Eastern Railway Leeds Extension between 1865 and 1869, to connect the Marsh Lane train station to the new Leeds central train station. It is comprised of a series of gently curving elevated brick masonry viaducts carrying two electrified rail tracks. Hundreds of passenger trains use these tracks every day, and many of the arches are tenanted.

This paper focusses on two brick arches on this viaduct, 37 and 38, shown in Fig. 1. These arches have a square span of $7.7 \mathrm{~m}$ and a width of $8 \mathrm{~m}$. The arch barrels are constructed of multiple rings with a total thickness of $\sim 0.5 \mathrm{~m}$ and a rise of $1.8 \mathrm{~m}$. The arch barrels are supported by approximately $0.9 \mathrm{~m}$ thick piers, which feature relieving 
arches (see Fig. 1b). Above the piers, it is thought that there is $1.15 \mathrm{~m}$ layer of backing, although detailed information on the materials and internal construction details of the bridge were not available.

Visible structural damage is evident in Arches 37 and 38. Key aspects of the damage is reported here, for more details refer to [1]. Significant transverse rotations are observed in the pier between 37 and 38, which are visible in Fig. 1b. Notably, the skewback stones decline in elevation on both sides of the relieving arch, resulting in a sagging springing line. Cracking in the relieving arch suggests an accompanying spreading mechanism where pier blocks to the left and right side of the relieving arch rotate in the transverse direction towards the spandrel walls. The longitudinal crack emanating from the relieving arch in Fig. 1b, is indicative of this. A seemingly independent transverse arch barrel crack was observed above the backing of the pier between Arches 37 and 38 (sketched in Fig. 1a). This crack resulted in significant water ingress and loss of mortar joints.

Various structural interventions were conducted to arrest the transverse movements observed in the arch barrel and piers. In the 1990s, ties were installed through the arch barrel to limit further opening of longitudinal barrel cracks (sketched in Fig. 1a). In September 2015, several additional ties were installed on the piers close to the ground level and on the spandrel walls. Furthermore, the relieving arch was tied internally with steel bars and filled with concrete. Some existing cracks were grouted and stitched.

Monitoring initiated after the completion of these strengthening activities in 2016. Initially, the research efforts focussed on understanding the underlying causes of existing damage in the viaduct. Then, detailed monitoring was conducted with innovative sensing techniques to obtain an improved understanding of the dynamic behavior. After identifying governing response mechanisms, a system for continuous monitoring of the asset was set up. This provided useful information on the propagation of damage and the effectiveness of the structural interventions.
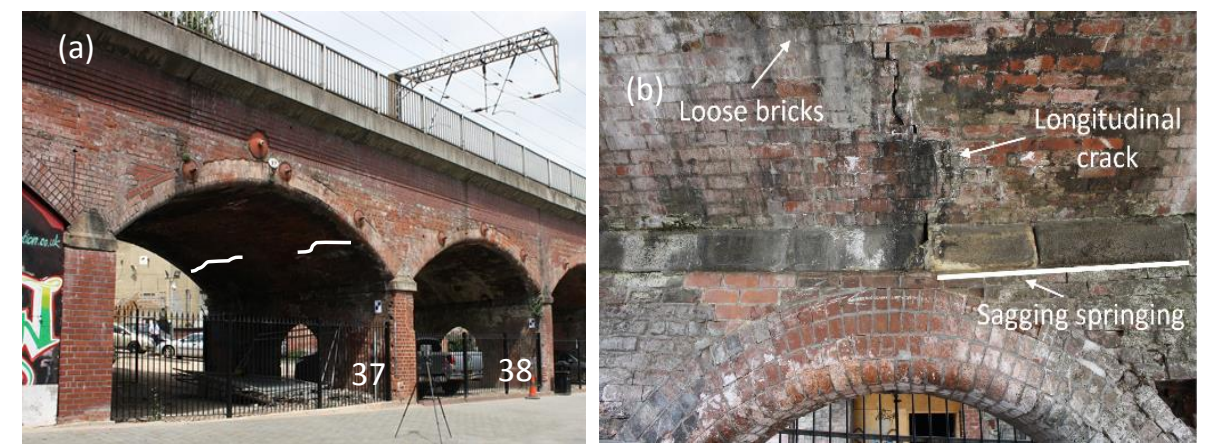

Fig. 1. (a) A side view of the Arches 37 and 38 of the Marsh Lane Viaduct and (b) a photo of the damage observed in the pier and Arch 38 barrel around the relieving arch located between Arches 37 and 38 . 


\section{Surveying, sensing and monitoring for structural assessment}

\subsection{Identifying reasons for existing damage}

Visual assessment reports dating back to 1970s indicate the presence of the aforementioned damage in Arches 37 and 38 and highlight growing concern due to increasing crack size and associated water damage. It is noteworthy that many other arches of the same viaduct exhibit similar signs of damage and different structural interventions were trialled to repair damage, with varying levels of success. Therefore, there is a need to understand the underlying causes for existing damage.

A new method to infer past support movements of the viaduct was proposed by [2]. To achieve this, the viaduct was surveyed with a laser scanner (see Fig. 1a) The gathered point clouds were registered to a common coordinate system, and segmented into smaller point clouds describing faces of the structural components, such as the arch barrel intrados. These point clouds were then fitted with primitive shapes, that represent the design intent. For instance, the arch intrados point cloud was fitted with a cylinder. This three-dimensional fit enabled the extraction of two dimensional arch slices across the square span, which were then fitted with circles.
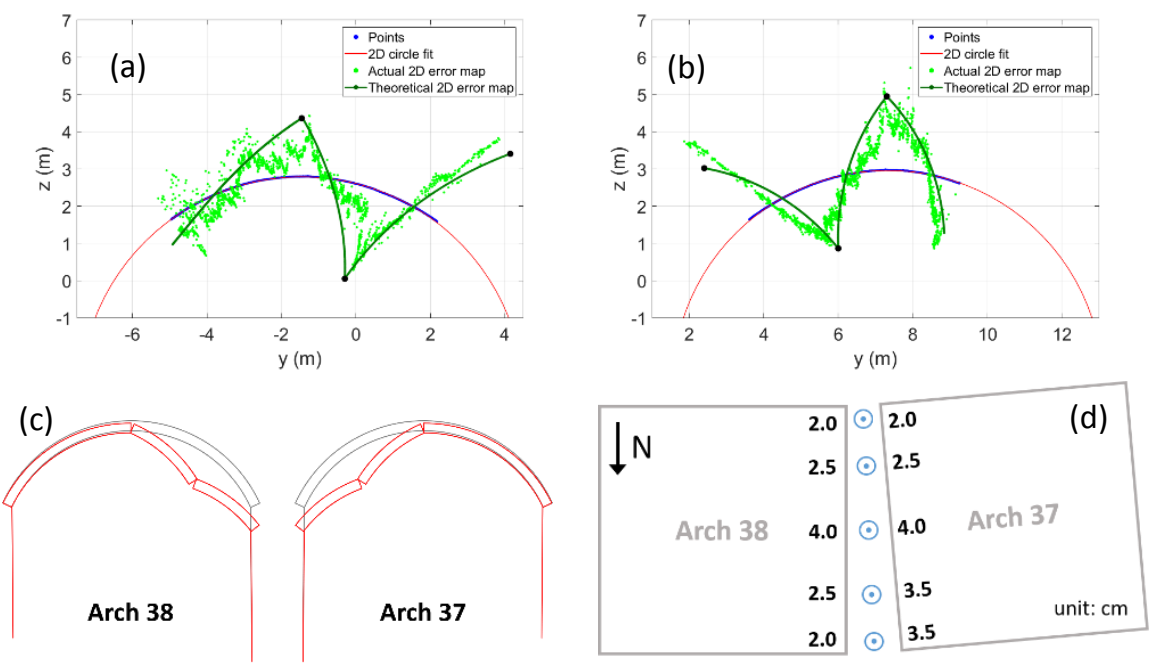

Fig. 2. Error map (x100 magnification) describing the discrepancy between the 2D point cloud slice and fitted circle for Arch (a) 38 and (b) 37. (c) The identified three hinge mechanism and support movement is illustrated with a schematic and (d) a plan view of the arches shows the relative settlement of the pier between Arch 37 and 38 identified from the analyses.

The discrepancies between the fitted shape and the actual point cloud is informative. This is shown in Fig. $2 \mathrm{a}$ and $\mathrm{b}$ for longitudinal arch slices passing through the centrelines of Arch 38 and 37. The real point cloud and the fitted circle are shown 
with blue dot markers and a red line in the background. In the foreground, green points demonstrate the discrepancy between the real point cloud and fitted shape. These error maps plot the discrepancies with 100 times magnification on the fitted shape. It is interesting to note that sharp kinks are observed in these error maps, and the distortions in Arches 38 and 37 look like mirror images of each other.

To understand the implications of these geometrical distortions, kinematic analyses were conducted on mechanisms of three hinged arches with various support movements. It was assumed that the kinks in the error maps describe hinges, where a rotational discontinuity is observed. Then, considering the mirror image symmetry in the Arch 38 and 37 error maps, it was postulated that a purely vertical support movement occurred at the pier between Arches 38 and $37.4 \mathrm{~cm}$ of vertical settlement at this pier, resulted in distorted theoretical arch shapes which were fitted with circles. Error maps associated to these theoretical distortions are shown in Fig. 2a and $2 \mathrm{~b}$ with black lines and demonstrate excellent agreement with the error maps. This indicates that $4 \mathrm{~cm}$ vertical support settlement of the pier at the centreline of the bridge (coinciding with the crown of the relieving arch) could explain the distortions in bridge geometry.

The kinematic mechanism and associated support movement identified using this approach are schematically shown in Fig. 2c. This mechanism indicates the presence of an extrados hinge near the quarter span of the arches. This is consistent with the presence of a transverse arch barrel crack which was discussed earlier (see Fig. 1a). Finally, Fig. 2d shows the settlements inferred from distorted arch barrel geometry across the width of the bridge. This analysis also clearly indicates that the settlements are the greatest at the bridge centreline, reducing in magnitude towards the spandrels.
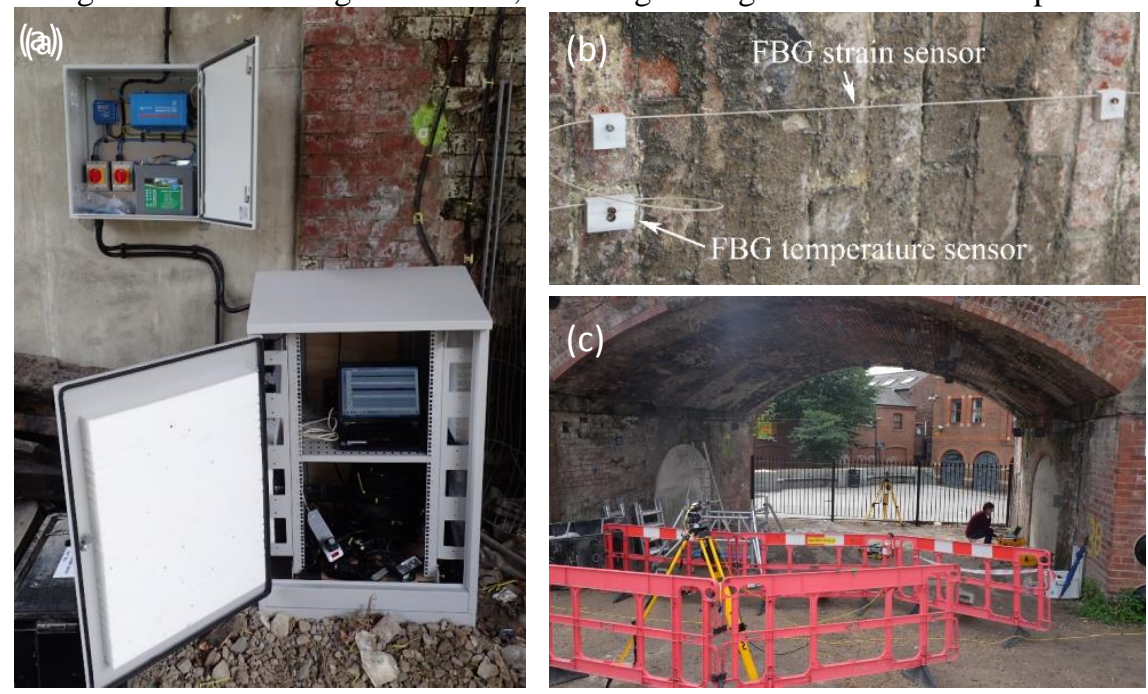

Fig. 3. (a) The fibre optic data acquisition system permanently installed under Arch 37, inside a temperature controlled cabinet, where it is connected to a laptop with a router, (b) FBG strain and temperature sensors, and attachment clamps, and (c) configuration of cameras for DIC monitoring. 


\subsection{Dynamic response of the damaged structure}

Quai-distributed monitoring techniques, such as sensing with Fibre Bragg Gratings (FBGs) and digital image correlation (DIC), make it feasible to obtain strain and displacement measurements across wide areas of the structure. FBG technology utilises a single mode silica fibre, along the length of which strain and temperature measurements can be conducted. DIC technique uses video data from commercial cameras to track small areas of the image with subpixel resolution. These tracking results can later be converted to displacement measurements [3]. Combined use of these techniques enable measurement of local (e.g. strain around cracks), and global (e.g. span opening and closing) quantities simultaneously.

FBGs were placed in the longitudinal and transverse directions over $\sim 1 \mathrm{~m}$ gauge lengths to measure dynamic strain. Temperature was measured and its effects on strain were compensated (Fig. 3b). Only a few measurements are discussed in this paper, detailed results can be found in [1]. Fibres strung across piers allowed measurement of span opening and closing, denoted by $\Delta$ (WP-EP). Strains across cracks in Arch 38 were monitored: $\varepsilon(\mathrm{A} 2-\mathrm{A} 3)$ measured longitudinal strains over the transverse crack while $\varepsilon(\mathrm{T} 4-\mathrm{T} 5)$ measured transverse strains at the tip of the longitudinal crack.

Meanwhile, the cameras were positioned centrally in line with the crown of the arches at a suitable distance (Fig. 3c). This setup allowed monitoring planar movements of Arches 37 and 38 in the vertical plane directly under the tracks, aligned with the bridge longitudinal axis. A useful measurement is the vertical crown displacement, $\Delta \mathrm{Y}(\mathrm{A} 5)$. Relative displacement measurements could also be obtained from the cameras, such as the measurement of span opening and closing, $\Delta$ (WP-EP), that could be directly compared to the FBG data.
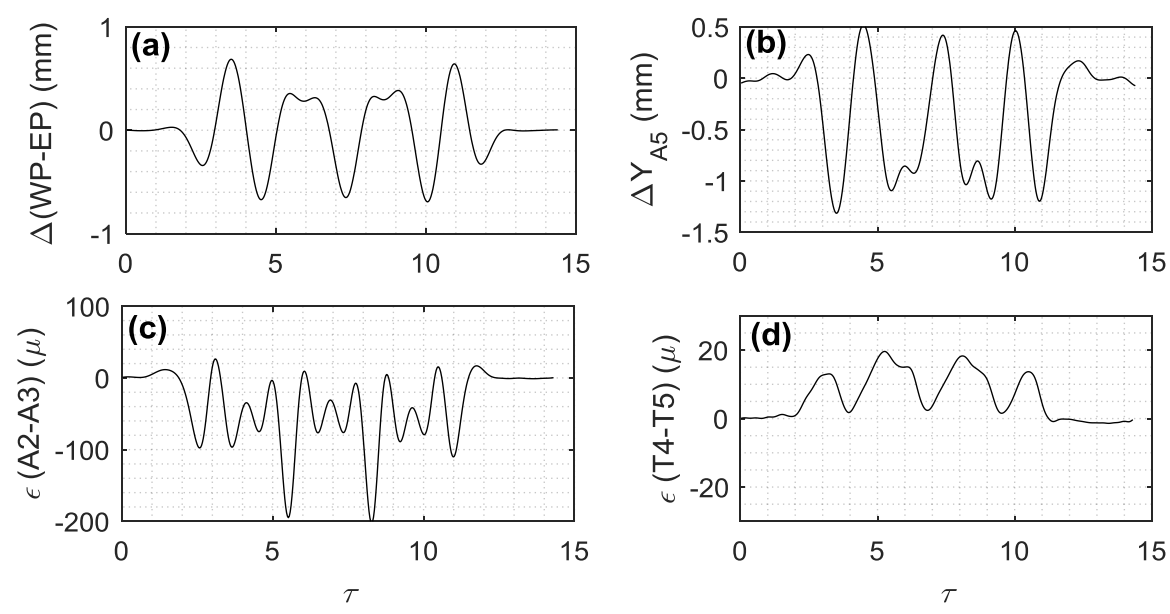

Fig. 4. (a) Span opening and closing, (b) crown vertical displacement, (c) longitudinal strain measurement over the transverse crack and (d) transverse strain measurement around the longitudinal crack tip during the passage of an eastbound Class 185 train on the northern side of Arch 38. 
Fig. 4 presents critical dynamic displacement and strain measurements recorded during the passage of a Class 185 train. In the $\mathrm{x}$-axis, $\tau$ is a dimensionless time parameter, normalized by train speed and arch span. The data is from underneath the northern tracks, where the eastbound train passes. Fig. 4 a shows the span opening and closing recorded by FBGs. This signal is influenced strongly by the relative position of axles and the span length of the arch. It is noteworthy that an identical maximum magnitude of $0.7 \mathrm{~mm}$ is observed for both span opening and closing. However, the accompanying vertical displacements at the crown (measured by DIC, see Fig. 4b) indicate different arch response mechanisms for span opening and closing. Crown descends by $1.3 \mathrm{~mm}$ when the span opens by $0.7 \mathrm{~mm}$, but it ascends only by $0.5 \mathrm{~mm}$ when the span closes by the same amount.

Fig. 4c shows the longitudinal strains experienced over the transverse crack sketched in Fig. 1a. The strains are compressive due to the closure of the open crack during train passage. It is noteworthy that the strains experienced at this location are an order of magnitude higher than all other locations. Fig. 4d shows the transverse strains experienced over the tip of the longitudinal crack visible in Fig. 4b. As expected, the strains are tensile, but strain magnitudes are very small.

Fig. 4 indicates significant response concentrated at areas of existing damage. To understand if structural degradation is ongoing, it is necessary to monitor structural response over longer periods and observe changes. This requires a low measurement noise (described as resolution) and high repeatability sensing system. Using the major peaks of span opening and closing signal observed during the passage of five similar eastbound Class 185 trains, the repeatability of FBG and DIC measurements were statistically evaluated (see Table 1). FBG relative displacement measurement resolution and repeatability are superior to DIC measurements, which are affected by numerous error sources [3]. Therefore, in order to monitor structural deterioration, it was decided to conduct further FBG strain measurements.

Table 1. Resolution and repeatability of span opening and closing measurements by FBG and DIC sensing techniques

\begin{tabular}{|c|c|c|}
\hline & Resolution $(\mathrm{mm})$ & Repeatability $(\mathrm{mm})$ \\
\hline$\Delta(\mathrm{WP}-\mathrm{EP})_{\mathrm{DIC}}$ & 0.091 & 0.082 \\
\hline$\Delta(\mathrm{WP}-\mathrm{EP})_{\mathrm{FBG}}$ & 0.006 & 0.026 \\
\hline
\end{tabular}

\subsection{Evaluation of ongoing deterioration}

[4] describes a solar panel powered Data Acquistion system (DAQ) that was permanently installed under Arch 37 inside a temperature controlled cabinet in July 2018 (see Fig. 3a). The DAQ features the fibre optic interrogator connected to a laptop with a router, which remotely transmits daily data through a $4 \mathrm{G}$ internet connection. A bespoke signal processing code was written to identify the type of train loading (train direction, velocity, number of carriages, relative axle distance) and determine statistical variations in dynamic strain under the same type of load.

Analyses of the response to eastbound Class 185 trains [5] have revealed that changes in temperature can cause variations up to $100 \%$ in dynamic strain measurements. For 
every Celsius degree increase, the mean value of the peak-to-peak amplitude of the signals decreases by $\sim 0.8 \mu \varepsilon$ for sensors in the longitudinal direction. In the transverse direction, the effects are less significant; for every $1{ }^{\circ} \mathrm{C}$ increase the mean value of the strain variation drops by $\sim 0.3 \mu \varepsilon$.

Thus, to investigate ongoing deterioration, it is informative to explore annual changes in data from the same calendar month, where temperature differences are negligible. Fig. 5 compares the mean and standard deviation values of the peak strain values of each sensor on the bridge gathered during November 2017 (black line) and November 2018 (grey line) under the Class-185 3-carriage train. All trains have similar speed and same direction to permit direct comparison. On the y-axis, solid lines show the mean values and the error bars indicate the standard deviation, $\pm \sigma$. On the $x$-axis of the plot, sensor ID's are given. For instance, sensor $38 \mathrm{~N}$ A2-A3 refers to the FBG gauge traversing the transverse crack in the north of the arch, as discussed in Fig. 4c. This sensor experiences the highest magnitude of strains. The locations of other listed sensors are described in [5].

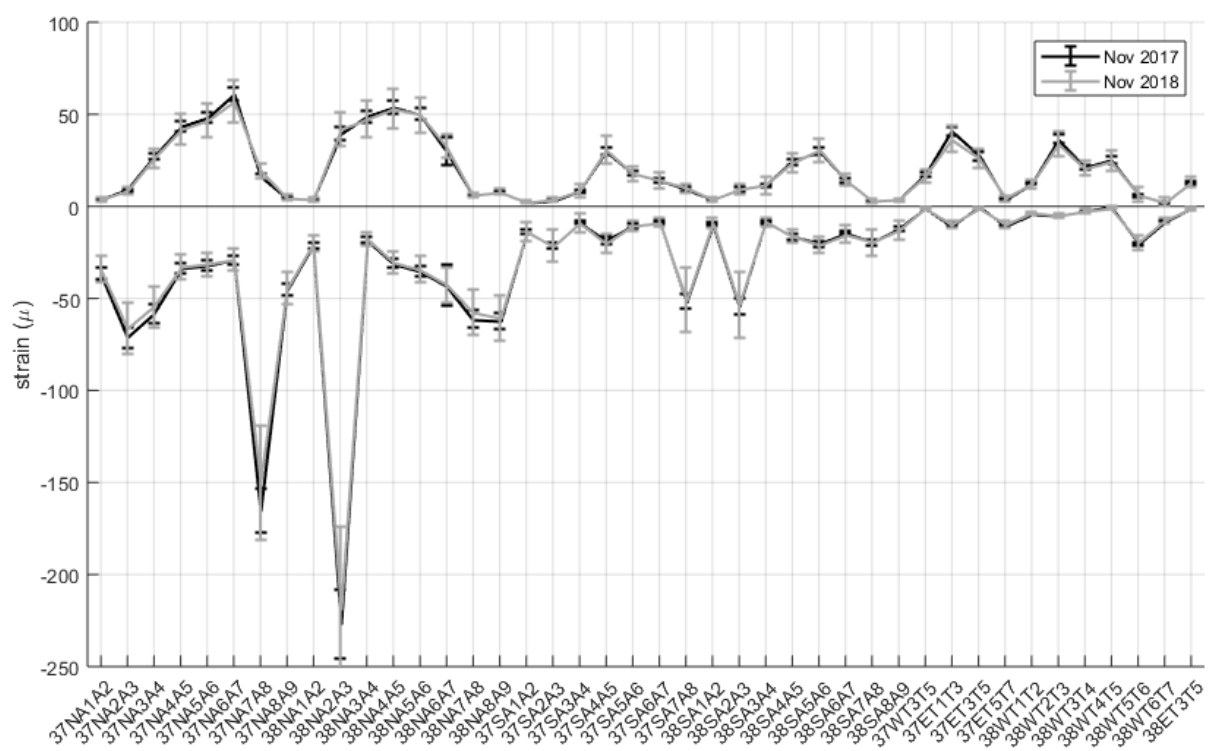

Fig. 5. (a) Span opening and closing, (b) crown vertical displacement, (c) longitudinal strain measurement over the transverse crack and (d) transverse strain measurement around the longitudinal crack tip during the passage of an eastbound Class 185 train on the northern side of Arch 38.

From Fig.5, it can be observed that the changes in the mean peak values of strain for all sensors are not statistically significant. The mean values and the standard deviations observed for all sensors are consistent. This indicates that noticeable progressive damage has not occurred in the bridge during the 1 year monitoring period. However, this is informative to explore past visual assessments, which indicate that the current damage occurred over periods of decade rather than one year. The fibre optic strain 
sensing technology allows strain measurements over such long time scales and can provide new insight into degradation behavior.

\section{Conclusions}

This paper discussed the results from a detailed monitoring campaign at the Marsh Lane viaduct in the UK. The monitoring campaign had the following objectives: (i) identify the reasons for existing damage, (ii) quantify their impact on the dynamic response of the structure and (iii) measure degradation of the response over a period of one year. These were achieved using new data analysis techniques with data from laser scanning, digital image correlation and fibre optic strain sensing.

One of the underlying reasons for existing damage in the bridge was identified by detailed analysis of arch barrel intrados distortions. These distortions demonstrated that transverse crack at the quarter span is acting as an extrados hinge, and that relative vertical settlement of one of the piers can sufficiently describe the observed distortions. This information was useful in the later evaluation of the dynamic response of the bridge using fibre optic strain sensing and digital image correlation based displacement monitoring. The data indicated the critical role of span opening and closing which governs behavior. These movements force the existing transverse crack to close, leading to large compressive strains. There are also accompanying tensile strains at the tip of the longitudinal crack, although the strain magnitudes are small.

Upon observing the high resolution and repeatability of fibre optic strain sensing, FBG sensors were permanently installed on the bridge to measure ongoing degradation. After removing the effects of temperature on dynamic strain measurements, no statistically significant changes were observed. This indicates that the structural interventions to arrest damage have been successful so far. Further monitoring (with fibre optics and acoustic emission sensors, see $[4,5]$ ) is currently underway to observe deterioration over longer durations and understand the mechanisms involved in this process.

\section{Acknowledgements}

This work is being funded by the Lloyd's Register Foundation, EPSRC and Innovate UK through the Data-Centric Engineering programme of the Alan Turing Institute and through the Cambridge Centre for Smart Infrastructure and Construction. Funding for the monitoring installation was provided by EPSRC under the Ref. EP/N021614/1 grant and by Innovate UK under the Ref. 920035 grant. The authors are grateful to Network Rail for providing power, technical support and site access for this research. 


\section{References}

1. Acikgoz, S., Kechavarzi, C., Soga, K., DeJong, M. J.: Dynamic response of a damaged masonry rail viaduct: Measurement and interpretation. Engineering Structures 168, 544558 (2018).

2. Ye, C., Acikgoz, S., Pendrigh, S., Riley, E., DeJong, M. J.: Mapping deformations and inferring movements of masonry arch bridges using point cloud data. Engineering Structures 173, 530-545 (2018).

3. Acikgoz, S., Soga, K., DeJong, M. J.: Sensing dynamic displacements in masonry rail bridges using 2D digital image correlation. Structural Control and Health Monitoring 25, e2187 (2018).

4. Alexakis, H., Franza, A., Acikgoz, S., DeJong, M. J.: A multi-sensing monitoring system to study deterioration of a railway bridge. In: Proceedings of the 9th International Conference on Structural Health Monitoring of Intelligent Infrastructure. St. Louis, Missouri, USA. August 4-7 (2019)

5. Alexakis, H., Franza, A., Acikgoz, S., DeJong, M. J.: Monitoring bridge degradation using dynamic strain, acoustic emission and environmental data. In: Proceedings of the 9th International Conference on Smart Infrastructure and Construction. Cambridge, UK. July 8$10(2019)$ 



\title{
The Marsh Lane Railway Viaduct: 2 years of monitoring with combined sensing and surveying technologies
}

\author{
Sinan Acikgoz ${ }^{1}$, Haris Alexakis ${ }^{2}$, Simon $_{\mathrm{Ye}^{2}}$, Andrea Franza ${ }^{3}$ and Matthew DeJong ${ }^{4}$ \\ ${ }^{1}$ University of Oxford, Oxford OX1 3TF, UK \\ ${ }^{2}$ University of Cambridge, Cambridge, CB2 1PZ, UK \\ ${ }^{3}$ Universidad Politécnica de Madrid, Madrid, 28040, Spain \\ ${ }^{2}$ University of California at Berkeley, Berkeley, 94720, CA, U.S.A. \\ sinan.acikgoz@eng.ox.ac.uk
}

Keywords: Masonry arch bridges, Point clouds, Primitive fitting, Fibre optic strain sensing, Digital image correlation

Marsh Lane viaduct is a typical example of a 19th century brick masonry railway arch in the UK [1]. It frequently carries passenger trains to and from Leeds Station. Visible structural damage is evident in Arches 37 and 38 which are explored in this paper. Significant transverse rotations are observed in the pier between 37 and 38 , especially around the relieving arch, resulting in a sagging springing line. A transverse arch barrel crack is also present above the backing, where water ingress and loss of mortar joints is evident. Recent structural interventions have seen filling of the relieving arch with reinforced concrete and the stitching of cracks to stop damage propagation. There is a need to understand the dynamic response of the structure after these interventions.

This paper broadly discusses the sensing techniques and associated analysis procedures used to (i) identify the reasons for existing damage, (ii) quantify their impact on the dynamic response of the structure and (iii) measure degradation of the response over a period of one year. To identify existing damage, distortions in geometry of the structure are examined with new point cloud processing techniques [2]. The distortions are identified with a respect to an ideal geometry represented by a primitive geometric shape (e.g. a cylinder). With the aid of kinematic analyses on three hinge arch mechanisms, these distortions are interpreted, and past support movements which may have caused the distortions are identified.

Then, to measure the dynamic response of the bridge, quasi-distributed fibre optic strain sensing and digital image correlation displacement measurement techniques are utilised. Combined use of these techniques enable measurement of local (e.g. strain around cracks), and global (e.g. span opening and closing) quantities simultaneously [3]. These measurements demonstrate the key role of span opening and closing, which governs behavior and imposes significant longitudinal compressive strains on the transverse crack. Small tensile strains are observed on the longitudinal crack tip. However, it is not clear if these measurements indicate damage propagation.

To track deterioration, a solar-powered data acquisition system was developed [4,5]. This enabled continuous fibre optic strain measurements between November 
2017 and 2018. These measurements revealed the important influence of temperature changes on peak dynamic strain amplitudes. After compensating for these effects, it was observed that the changes in mechanical response of the structure during the one year monitoring period was not statistically significant. Further monitoring (with fibre optics and acoustic emission sensors) is currently underway to observe deterioration over longer durations and understand the mechanisms involved in this process.

\section{References}

1. Acikgoz, S., Kechavarzi, C., Soga, K., DeJong, M. J.: Dynamic response of a damaged masonry rail viaduct: Measurement and interpretation. Engineering Structures 168, 544558 (2018).

2. Ye, C., Acikgoz, S., Pendrigh, S., Riley, E., DeJong, M. J.: Mapping deformations and inferring movements of masonry arch bridges using point cloud data. Engineering Structures 173, 530-545 (2018).

3. Acikgoz, S., Soga, K., DeJong, M. J.: Sensing dynamic displacements in masonry rail bridges using 2D digital image correlation. Structural Control and Health Monitoring 25, e2187 (2018).

4. Alexakis, H., Franza, A., Acikgoz, S., DeJong, M. J.: A multi-sensing monitoring system to study deterioration of a railway bridge. In: Proceedings of the 9th International Conference on Structural Health Monitoring of Intelligent Infrastructure. St. Louis, Missouri, USA. August 4-7 (2019)

5. Alexakis, H., Franza, A., Acikgoz, S., DeJong, M. J.: Monitoring bridge degradation using dynamic strain, acoustic emission and environmental data. In: Proceedings of the 9th International Conference on Smart Infrastructure and Construction. Cambridge, UK. July 810 (2019) 